\title{
Potential Antioxidant and Wound Healing Effect of Nano-Liposol with High Loading Amount of Astaxanthin
}

This article was published in the following Dove Press journal: International Journal of Nanomedicine

\author{
Hyeryeon $\mathrm{Oh}^{1,2}$ \\ Jin Sil Lee ${ }^{1,2}$ \\ Daekyung Sung' \\ Jong-Min $\operatorname{Lim} \mathbb{D}^{3,4}$ \\ Won II Choi (D)
}

'Center for Convergence Bioceramic Materials, Convergence R\&D Division, Korea Institute of Ceramic Engineering and Technology, Cheongju, Chungbuk 28160, Republic of Korea; ${ }^{2}$ School of Materials Science and Engineering and Department of Nanobio Materials and Electronics, Gwangju Institute of Science and Technology, Gwangju 500-7I2, Republic of Korea; ${ }^{3}$ Department of Chemical Engineering, Soonchunhyang University, Asan, Chungnam 31538, Republic of Korea; ${ }^{4}$ Department of Electronic Materials and Devices Engineering, Soonchunhyang University, Asan, Chungnam 31538, Republic of Korea

\begin{abstract}
Background: Astaxanthin (ASTA), a carotenoid, is a strong antioxidant. However, its application in functional foods, pharmaceuticals, and cosmetics remains limited due to its low aqueous solubility and stability. Several different encapsulating materials have been used to improve the stability and bioavailability of ASTA; however, the currently investigated nano-carriers for ASTA require additional improvements with regard to their loading capacity and stability.
\end{abstract}

Methods: In this study, we developed lecithin nano-liposol (Lec NS) as a novel carrier of ASTA using a simple emulsion evaporation method. The physicochemical characteristics including hydrodynamic diameter, polydispersity index, surface charge and morphology were analyzed by DLS and TEM. The antioxidant activity of the ASTA-loaded Lec NS (ASTA@Lec NS) was evaluated using a DPPH radical scavenging assay and in vitro antioxidant assay. The study of in vitro wound healing efficacy was carried out to observe the beneficial effect of antioxidant activity of ASTA@Lec NS on cell migration.

Results: ASTA@Lec NS showed improved stability and efficacy owing to improved aqueous solubility of ASTA inside Lec NS. Both in situ and in vitro antioxidant activities of ASTA@Lec NS were higher than that of bare ASTA and Lec NS. It also exhibited strong wound healing efficacy by regulation of ROS level in in vitro cell model.

Conclusion: This study revealed that the encapsulation of ASTA into Lec NS using a wet phase transfer enhanced its physiological stability and bioavailability for effective scavenging of reactive oxygen species.

Keywords: optimization, lipid micelle, reactive oxygen species, ROS scavenging, cell proliferation

\section{Introduction}

Astaxanthin (ASTA) is a natural carotenoid found mostly in yeast, algae, and other aquatic species. ${ }^{1}$ Like most carotenoids containing long chains of conjugated polyenes, ASTA is red in color and possesses high antioxidant activity. ${ }^{2}$ Its strong antioxidative properties are attributed to the hydroxyl and keto groups at the ends of its molecular structure making it more antioxidative than other carotenoids or even the well-known lipophilic antioxidant $\alpha$-tocopherol. ${ }^{3}$ Multiple studies have found ASTA to be potentially useful as a nutraceutical and cosmeceutical for treating various diseases mediated by oxidative stress. ${ }^{4-6}$ Oxidative stress is caused by an excess generation of reactive oxygen species (ROS) that damage biomolecules and lead to cell death. ROS are derived from oxygen as highly reactive forms of superoxide $\left(\mathrm{O}_{2}{ }^{-}\right)$, hydroxyl radical

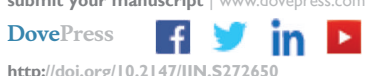

International Journal of Nanomedicine 2020:15 923I-9240

9231

(c) (i) (c) 2020 oh et al. This work is published and licensed by Dove Medical Press Limited. The full terms of this license are available at https://www.dovepress.com/terms.php C. ${ }_{\mathrm{BY}} \mathrm{NC}$ and incorporate the Creative Commons Attribution - Non Commercial (unported, v3.0) License (http://creativecommons.org/licenses/by-nc/3.0/). By accessing the work you hereby accept the Terms. Non-commercial uses of the work are permitted without any further permission from Dove Medical Press Limited, provided the work is properly attributed. For permission for commercial use of this work, please see paragraphs 4.2 and 5 of our Terms (https://www.dovepress.com/terms.php). 
$(\mathrm{OH} \bullet)$, hydrogen peroxide $\left(\mathrm{H}_{2} \mathrm{O}_{2}\right)$, and hypochlorite $\left(\mathrm{ClO}^{-}\right)$. It has been determined that ASTA scavenges ROS through several assays including 2,2-diphenyl-1-picrylhydrazyl (DPPH), superoxide anion, and hydroxyl radicals ${ }^{7}$ and thereby has a protective effect against various diseases. ${ }^{8-10}$ Regulation of optimal ROS levels also plays an important role in wound healing, while several studies have reported wound healing activity of ASTA. ${ }^{11-13}$ ASTA is involved in processes such as the expression of growth factors, cellular differentiation, angiogenesis, wound healing, and repair, and inflammation. ${ }^{14,15}$

While it exhibits antioxidative activity, highly unsaturated ASTA is susceptible to heat, light, oxygen, and other external factors. ${ }^{16}$ Therefore, ASTA is easily degraded during its preparation and storage. Furthermore, it has low bioavailability for in vivo application due to its lipophilicity. ${ }^{17}$ To counteract these problems, ASTA has been encapsulated using various materials and is typically stored in powder form. It has been reported that complexation of ASTA improves its stability and shelf-life of bioactivity. ${ }^{18}$ The use of polymers, ${ }^{19,20}$ proteins, ${ }^{21}$ and carbohydrates such as cyclodextrin ${ }^{22,23}$ have all been shown to possibly improve the stability, water-dispersibility, and bioavailability of ASTA. Amphiphilic liposomes are especially capable of loading fat-soluble ASTA into their lipophilic cores, but have low loading capacities. ${ }^{24,25}$ In addition, several studies have developed ASTA-encapsulated nano-carriers, but these have been primarily prepared using additional emulsifiers such as polysorbate 20 , polysorbate 80 , cholesterol, and others. ${ }^{26,27}$ Despite the use of these carriers, the currently available delivery systems of ASTA still require improvement in both their loading capacity and stability.

Therefore, in this study, we developed a lecithin nanoliposol (Lec NS) carrier loaded with ASTA (ASTA@Lec NS) using a simple method of phase transfer in order to enhance ASTA stability and bioavailability (Figure 1A). Dispersion of ASTA in aqueous solution using the Lec NS improves its efficacy and possibly broaden its application in various fields. Through formulation optimization, the Lec NS exhibited a high loading capacity of ASTA. Physicochemical characteristics such as size, polydispersity, surface charges, and morphology of the nano-liposol were analyzed using dynamic light scattering (DLS), ultraviolet-visible (UV-Vis) spectroscopy, and transmission electron microscopy (TEM). The ASTA@Lec NS was highly stable at $37^{\circ} \mathrm{C}$ and showed enhanced antioxidant activity and wound healing efficacy in vitro.
A
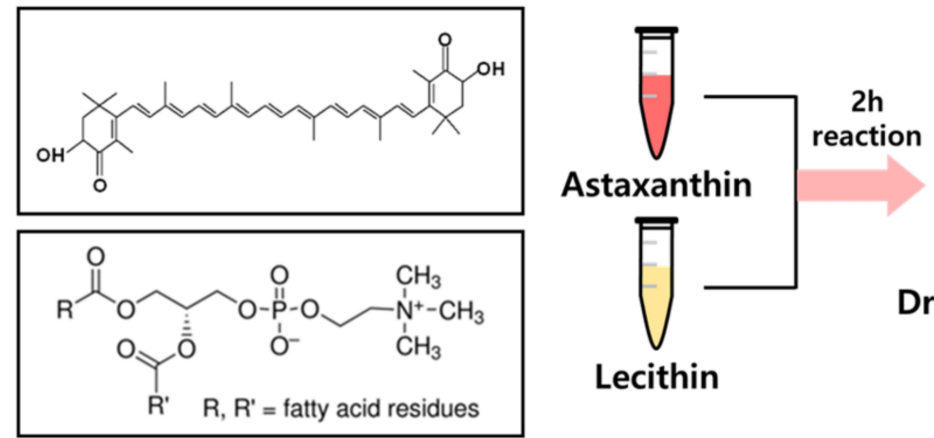

Homogenization Ultrasonication
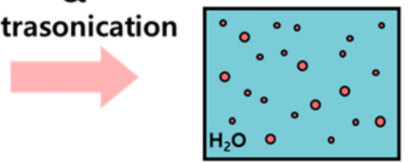

Dropwise to DIW

ASTA@Lec NS

\section{B}

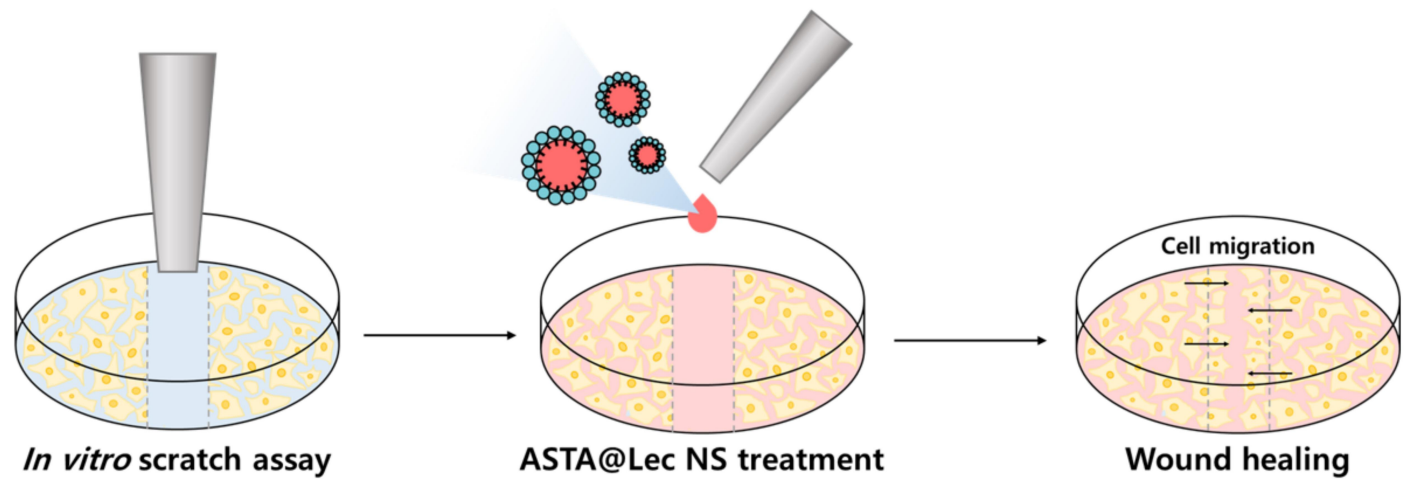

Figure I Development of astaxanthin-loaded lecithin nano-liposol (ASTA@Lec NS). (A) Preparation scheme of ASTA@Lec NS. (B) Scratch wound healing assay of ASTA@Lec NS following scratch wound made by a sterile p1000 micropipette tip across the NIH 3T3 fibroblast monolayer.

Abbreviations: DIW, deionized water; ASTA@Lec NS, astaxanthin-loaded lecithin nano-liposol. 


\section{Materials and Methods \\ Materials}

ASTA was purchased from Sigma-Aldrich (St. Louis, MO, USA) and GDE Ltd. (Siheung, Korea). L- $\alpha$ phosphatidylcholine from soybean (lecithin), sucrose, and DPPH were purchased from Sigma-Aldrich. $\mathrm{H}_{2} \mathrm{O}_{2}(30 \%)$ was obtained from Junsei Chemical Co. (Tokyo, Japan). Chloroform was obtained from Wako Chemicals Ltd (Osaka, Japan) and methanol was purchased from SK Chemical (Seongnam, Korea). Deionized (DI) water and phosphate-buffered saline (PBS) were obtained from HyClone (Logan, UT, USA). The murine fibroblast NIH 3 T3 cells (ATCC, MD, USA) used in the in vitro assays were cultured in Dulbecco's modified Eagle's medium (DMEM; Gibco, Grand Island, NY, USA) supplemented with fetal bovine serum (FBS) from Gibco and antibioticantimycotic (AA) from Thermo Fisher Scientific (Waltham, MA, USA). An in vitro indicator of ROS, 2' $7^{\prime}$ dichlorodihydrofluorescein diacetate (H2DCFDA), was purchased from Invitrogen (Carlsbad, CA, USA).

\section{Preparation of Astaxanthin-Loaded Lecithin Nano-Liposol (ASTA@Lec NS)}

Lec NS with various concentrations of ASTA loaded was simply prepared using an emulsion evaporation method with modification. ${ }^{28}$ Briefly, $1 \mathrm{~mL}$ chloroform solution containing various concentrations of ASTA $(0,0.4,1,2$, 3,4 , and $10 \mathrm{mg} / \mathrm{mL}$ ) was added to $20 \mathrm{mg}$ of lecithin. After $2 \mathrm{~h}$ of mixing using a rotatory shaker, each mixture was slowly added to $5 \mathrm{~mL}$ DI water with gentle stirring and homogenized for $5 \mathrm{~min}$ at $5000 \mathrm{rpm}$. The chloroform solvent was then removed by vacuum drying for $3 \mathrm{~h}$ and the remaining solution was subjected to ultrasonication (amplitude $=20 \%$, on $/$ off time $=5 \mathrm{~s} / 15 \mathrm{~s}$ ) for $20 \mathrm{~min}$. Next, the ASTA@Lec NS was purified by centrifugation at $2000 \mathrm{rpm}$ for $15 \mathrm{~min}$ using an Amicon Ultra-15 centrifugal filter (molecular weight cut-off $100 \mathrm{kDa}$; Merck Millipore, Burlington, MA, USA) to remove unloaded ASTA and then filtered through a sterile $0.45 \mu \mathrm{m}$ syringe filter.

\section{Characterization of ASTA@Lec NS}

The hydrodynamic diameter, polydispersity index (PDI), and zeta potential of ASTA@Lec NSs with different loaded ASTA content were characterized by DLS measurements using a Zetasizer instrument (ELSZ-2000,
Otsuka, Osaka, Japan). Loading content (LC) of ASTA was determined using the following equation: ${ }^{29}$

$$
\begin{aligned}
& \mathrm{LC}(\mathrm{wt} \%) \\
& =\left[\frac{\left(\begin{array}{ll}
\text { weight of feeding } & \text { weight of } \\
\text { ASTA } & \text { unloaded ASTA }
\end{array}\right)}{\text { weight of LecNS }}\right]
\end{aligned}
$$

Loading efficiency (LE) of ASTA into the Lec NS was calculated using the following equation:

$$
\begin{aligned}
& \text { LE }(\%)
\end{aligned}
$$

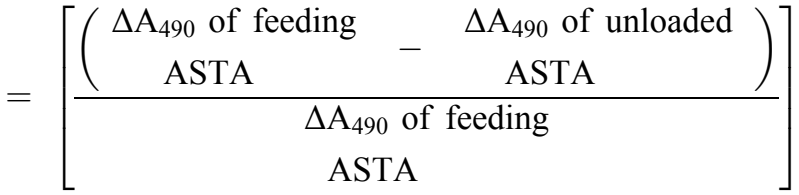

where $\Delta \mathrm{A}_{490}$ of feeding ASTA and unloaded ASTA were the UV-Vis absorbance of the initial ASTA and remaining ASTA after encapsulation at a wavelength of $490 \mathrm{~nm}^{30}$ The amount of ASTA in the Lec NS was quantified via a chemical colorimetric technique using a UV-Vis spectrophotometer (Mega900, Scinco, Seoul, Korea). Morphology of the ASTA@Lec NS was analyzed by TEM by evaluating $20 \mu \mathrm{L}$ of sample on a copper grid with 200 mesh carbon film using a JEM-2100Plus HR (JEOL, Tokyo, Japan).

\section{In situ Antioxidant Activity of ASTA@Lec NS}

Antioxidant activities of ASTA and ASTA@Lec NS were evaluated using the stable-free radical $\mathrm{DPPH}^{31}$ Bare ASTA and ASTA@Lec NS (LC 15 wt \%) were dissolved in DI water at various concentrations. DPPH solution (0.5 $\mathrm{mM}$ ) was prepared in methanol. Then, $150 \mu \mathrm{L}$ of each sample solution and $150 \mu \mathrm{L}$ of DPPH solution were combined in a 96-well plate. To prepare the negative control, $150 \mu \mathrm{L}$ of DI water was added to the DPPH solution instead of sample solution. Following a 30 min incubation with minimal exposure of light, absorbance of the reaction mixtures was measured at a wavelength of $515 \mathrm{~nm}$ using a microplate reader (VICTOR X5, PerkinElmer, Singapore, Republic of Singapore). Antioxidant activity was calculated using the following equation:

$$
\begin{aligned}
& \text { Antioxidant activity }(\%) \\
& =\left[\frac{\left(\Delta \mathrm{A}_{515} \text { of control }-\Delta \mathrm{A}_{515} \text { of sample }\right)}{\Delta \mathrm{A}_{515} \text { of control }}\right]
\end{aligned}
$$


As an overlap of absorbance at $515 \mathrm{~nm}$ with the sample absorbance may lead to inaccurate results, ${ }^{32}$ background absorbance was subtracted from the absorbance of the test samples.

\section{Stability of ASTA@Lec NS}

To increase the storage stability of ASTA@Lec NS, the Lec NS was freeze-dried with different amounts of sucrose as a cryoprotectant $(0,1,5,10$, and $15 \mathrm{wt} \%)$ for $3 \mathrm{~d} .{ }^{33}$ The appearance after lyophilization with sucrose was observed and stability of the physicochemical characteristics of the ASTA@Lec NS was also analyzed. Lyophilized nanoliposols were dissolved in biological buffer (PBS) and then kept at room temperature or in a shaking incubator (100 rpm) at $37^{\circ} \mathrm{C}$. The samples were monitored for 4 weeks for any change in dispersity or hydrodynamic diameter.

\section{In vitro Cytotoxicity of ASTA@Lec NS}

For in vitro assays, NIH 3T3 cells were cultured in DMEM containing 10\% FBS and 1\% AA. To evaluate cytotoxicity of Lec NS and ASTA@Lec NS, cells were seeded into a 96-well plate at a density of 10,000 cells per well and incubated in a humidified atmosphere of $5 \% \mathrm{CO}_{2}$ at $37^{\circ} \mathrm{C}{ }^{34,35}$ After $12 \mathrm{~h}$ incubation, different concentrations of the nano-liposols were added to the cells and incubation continued for $24 \mathrm{~h}$. Then, Cell-Counting Kit-8 (CCK-8) solution (Dojindo Laboratories, Japan) diluted to $10 \%$ in DMEM was added to each well for $1 \mathrm{~h}$. Viable cells produced orange-colored formazan at an absorbance of $450 \mathrm{~nm}$. The absorbance was measured using a microplate reader and cell viability calculated using the following equation:

Cell viability $(\%)=\left(\frac{\Delta \mathrm{A}_{450} \text { of test group }}{\Delta \mathrm{A}_{450} \text { of control group }}\right) \times 100$

\section{In vitro Antioxidant Activity of ASTA@Lec NS}

The in vitro ability to scavenge ROS generated from NIH $3 \mathrm{~T} 3$ cells stimulated with the oxidative stress agent $\mathrm{H}_{2} \mathrm{O}_{2}$ was analyzed according to a method previously described. ${ }^{36}$ Briefly, the NIH $3 \mathrm{~T} 3$ fibroblast cells were seeded into a 96-well plate (10,000 cells/well). Following an overnight incubation, cells were treated with Lec NS or ASTA@Lec NS $(10 \mu \mathrm{g} / \mathrm{mL})$ and then subsequently treated with $\mathrm{H}_{2} \mathrm{O}_{2}(5 \mu \mathrm{M})$ for $8 \mathrm{~h}$, and were then washed with PBS to remove the remaining media. H2DCFDA solution (10 $\mu \mathrm{M})$ was added to each well for 90 min under dark conditions. In the presence of ROS, the detection reagent was oxidized to fluorescent dichlorofluorescein (DCF). Thus, the in vitro antioxidant activity of ASTA@Lec NS could be measured using a microplate reader according to the decrease in fluorescence at an excitation wavelength of $485 \mathrm{~nm}$ and an emission wavelength of $535 \mathrm{~nm}$.

\section{In vitro Wound Healing Effect of ASTA@Lec NS}

A scratch wound-healing assay was performed as previously reported to evaluate enhanced migration of fibroblast cells following treatment of ASTA@Lec NS (Figure 1B). ${ }^{37}$ First, cells were seeded into a 24 -well plate $(200,000$ cells/well) and grown to confluency. A scratch wound was then made using a sterile P1000 micropipette tip. Cell debris was removed by washing with DMEM and different concentrations of ASTA@Lec NS were used to treat the cells. The control group received only DMEM without FBS. Wound closure was evaluated at each desired time point over a 24-h incubation period using a DMil light microscope (Leica, Wetzlar, Germany) at $4 \times$ magnification. The wound gap distance was calculated by using ImageJ software 1.8.0 (NIH, Bethesda, MD, USA).

\section{Statistical Analysis}

All experiments were performed in triplicate and the resulting data are shown as the mean \pm standard deviation. Experimental groups were statistically compared using Student's $t$-test. Differences were considered to be statistically significant or highly significant at $\mathrm{p}$-values $<0.05$ or $<0.001$, respectively.

\section{Results and Discussion}

\section{Preparation and Characterization of ASTA@Lec NS}

ASTA@Lec NS containing various concentrations of ASTA were prepared through reactions using ASTA and lecithin in the chloroform solvent. Oil-in-water ASTA emulsions were formed by adding the mixtures to the water phase and the nanoscale preparations generated by homogenization and ultrasonication following the removal of organic solvent. As the ASTA@Lec NS developed, the nano-liposols exhibited red color with increasing loading contents of ASTA (Figure 2A). The hydrodynamic diameter prior to loading was $140 \pm 4 \mathrm{~nm}$, which was consistently maintained, even after loading up to $15 \mathrm{wt} \%$ 
A

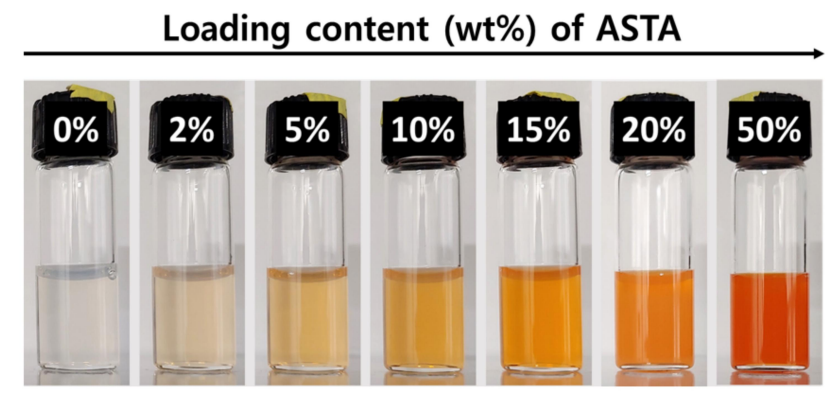

C

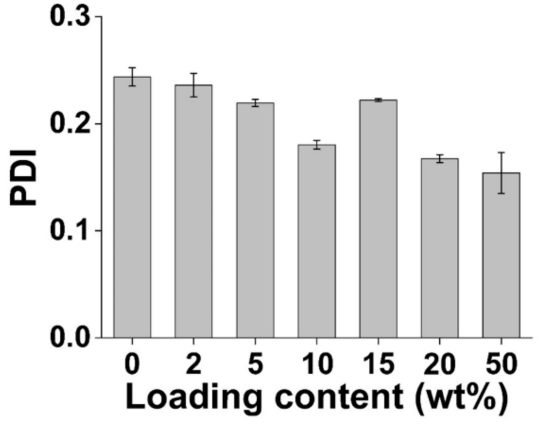

D

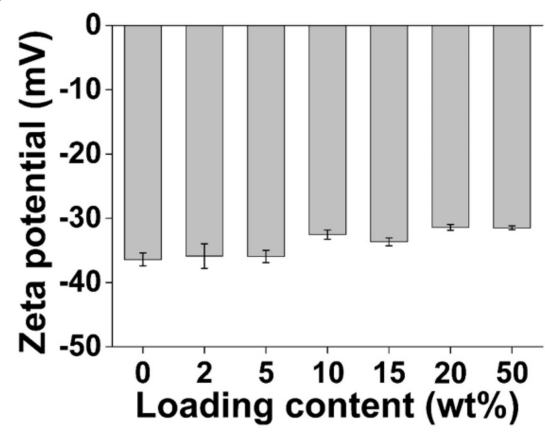

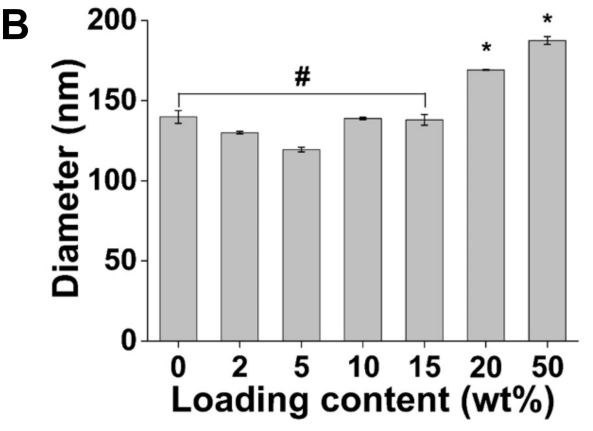

E

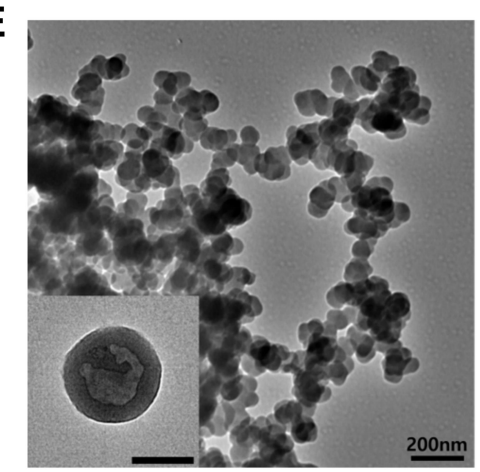

Figure 2 Characterization of astaxanthin-loaded lecithin nano-liposol (ASTA@Lec NS). (A) Loading content; (B) hydrodynamic diameters; (C) polydispersity index (PDI); (D) zeta potential of ASTA@Lec NS with different loading contents of astaxanthin (ASTA). (E) Transmission electron microscopy (TEM) image of ASTA@Lec NS with a loading content of $15 \mathrm{wt} \%$ (scale bar $=200 \mathrm{~nm}$ ).

Notes: ${ }^{\#}$ or * refers p-value above 0.05 or below 0.05 , respectively.

Abbreviations: ASTA, astaxanthin; wt $\%$, weight percent; PDI, polydispersity index.

ASTA (Figure 2B). However, the ASTA@Lec NSs with loading contents of 20 and $50 \mathrm{wt} \%$ had larger diameters of $169 \pm 1 \mathrm{~nm}$ and $187 \pm 2 \mathrm{~nm}$, respectively, indicating that the ASTA was overloaded $(\mathrm{p}<0.05)$. There was no significant change in PDI or zeta potential (Figure $2 \mathrm{C}$ and $\mathrm{D}$ ). The nano-liposols were well dispersed with PDI values below 0.3 while their surfaces were negatively charged with zeta potentials of approximately $-33.9 \pm 0.9 \mathrm{mV}$. The morphology of the nano-liposols was evaluated by TEM (Figure 2E). As expected from the DLS analysis data, the ASTA@Lec NS (15 wt\%) were spherical in shape of similar size (approximately $150 \mathrm{~nm}$ ). Thus, it was determined that the optimal loading capacity of ASTA@Lec NS was 15 wt $\%$ with an entrapment efficiency of $98.8 \%$. Subsequent experiments were performed using ASTA@Lec NS containing 15 wt $\%$ ASTA.

\section{In situ Antioxidant Activity of ASTA@Lec NS}

Antioxidant activity of ASTA@Lec NS was evaluated according to its ability to serve as an electron donor where deep violet DPPH radicals are decolorized and become yellow. Consistent with Li et al, ${ }^{38}$ ASTA was more effective at radical scavenging

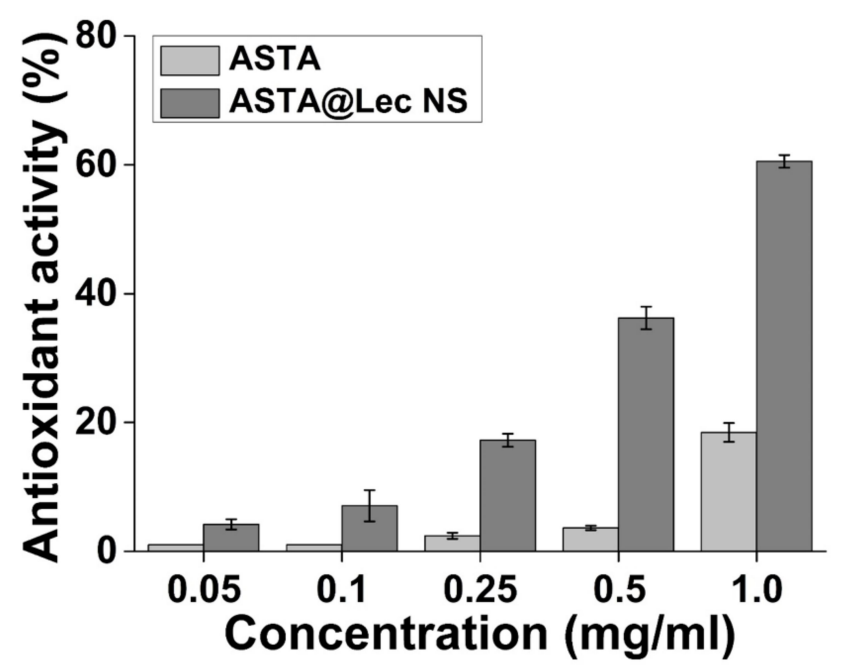

Figure 3 In situ antioxidant activity analysis of astaxanthin (ASTA) and astaxanthinloaded lecithin nano-liposol (ASTA@Lec NS; 15 wt\%) at various concentrations of ASTA according to a 2.2-diphenyl-I-picrylhydrazyl-free radical scavenging assay. Abbreviations: ASTA, astaxanthin; ASTA@Lec NS, astaxanthin-loaded lecithin nano-liposol.

at higher concentrations (Figure 3). However, much higher concentrations of free ASTA were required to reach approximately $20 \%$ antioxidant activity as an aqueous solution of ASTA was used for the DPPH radical scavenging assays in 
this study instead of an organic solvent. This suggests that the solubilization of ASTA is important for its radical scavenging activity. For this reason, the antioxidant activity of ASTA improved significantly following encapsulation in Lec NS. At the same concentration of $1 \mathrm{mg} / \mathrm{mL}$, the antioxidant activity of ASTA@Lec NS was approximately 60\%, which was much higher than that of bare ASTA. This result was consistent with a previous report. ${ }^{30}$ Furthermore, it is worth noting that the use of high amounts of encapsulating material decreased the antioxidant activity of the complexes by reducing the accessibility of ASTA to radicals, which was previously reported by Yuan et al. $^{23}$

\section{Stability Testing of ASTA@Lec NS}

The ASTA@Lec NS was prepared as a powder by lyophilization to assure extended maintenance of ASTA bioactivity. To prevent agglomeration of the nano-liposols during lyophilization, the amount of sucrose used as a cryoprotectant was optimized. Without sucrose, the diameter of the nano-liposols increased to $644 \pm 16 \mathrm{~nm}$ after freeze drying (Figure 4A). The addition of more than $5 \mathrm{wt} \%$ of sucrose kept the diameter of the nano-liposols similar to that prior to freeze drying. With increasing amounts of sucrose, the PDI values decreased below 0.3 and the zeta potential of the nano-liposols became less negative (Figure 4B and C). Furthermore, it was easier to obtain a fine powder of ASTA@Lec NS with more than 10 wt $\%$ sucrose, suggesting that $10 \mathrm{wt} \%$ sucrose was optimal for freeze drying ASTA@Lec NS (Figure 4D). This was consistent with a previous report that sucrose enhances storage stability post lyophilization by lowering residual water content. $^{39}$ Therefore, samples lyophilized with insufficient amounts of sucrose $(<5 \mathrm{wt} \%)$ were too sticky to be ground and considered unsuitable for subsequent applications.

The stability of ASTA@Lec NS in solution was also evaluated for 4 weeks at $25^{\circ} \mathrm{C}$ and $37^{\circ} \mathrm{C}$ (Figure 5). The diameters and PDIs of ASTA@Lec NS barely changed, even after prolonged storage at $25^{\circ} \mathrm{C}$ and $37^{\circ} \mathrm{C}$, indicating that the nano-liposols could maintain their nanostructure for sufficiently long periods of time at both room temperature and body temperature.

\section{In vitro Cytotoxicity and Antioxidant Activity of ASTA@Lec NS}

CCK assays were used to evaluate the viability of murine dermal fibroblasts following treatment with Lec NS and ASTA@Lec NS (Figure 6A). Both nano-liposols with and without ASTA demonstrated no cytotoxicity, resulting in $>95 \%$ viability at a concentration of $1000 \mu \mathrm{g} / \mathrm{mL}$. In addition, Y. Chen et al found using MTT assays that ASTA does not affect the proliferation of human dermal fibroblast. ${ }^{8}$ This
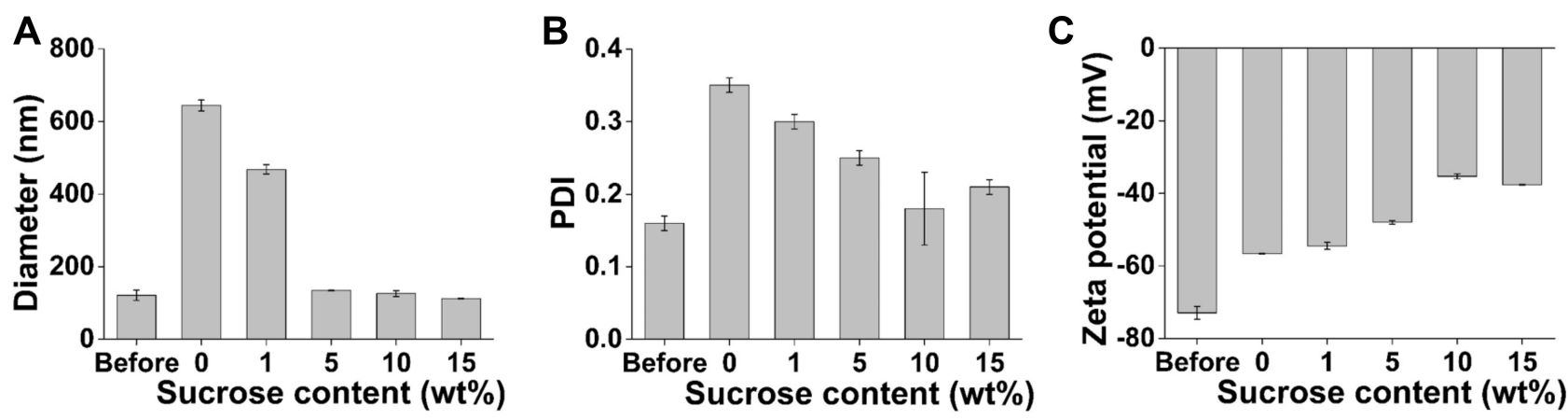

D

Sucrose content (wt.\%)

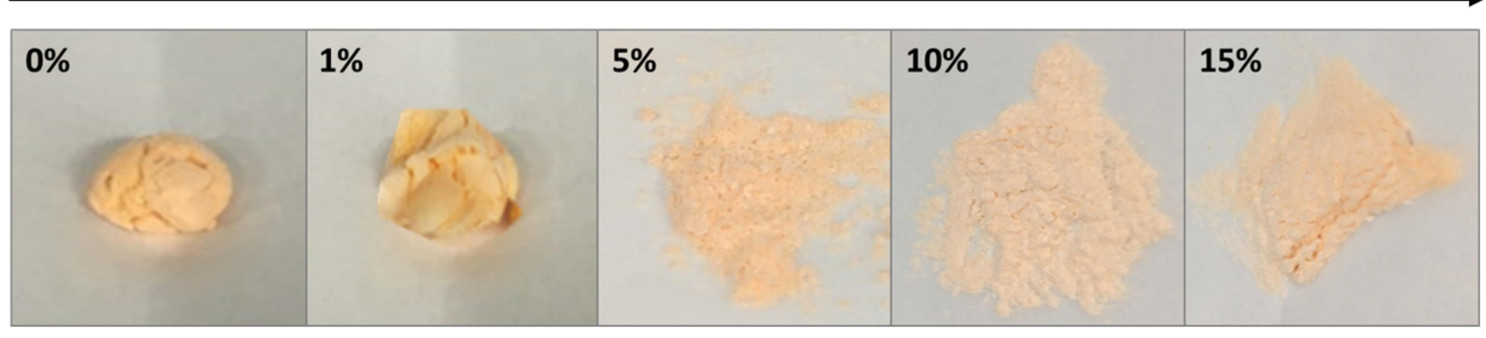

Figure 4 Stability of astaxanthin-loaded lecithin nano-liposol (ASTA@Lec NS; 15 wt\%) following lyophilization using sucrose as a cryoprotectant. (A) Hydrodynamic diameter; (B) polydispersity index (PDI); (C) zeta potential; (D) photographs of ASTA@Lec NS (I5 wt\%) following lyophilization using different amounts of sucrose. Abbreviations: wt\%, weight percent; PDI, polydispersity index. 

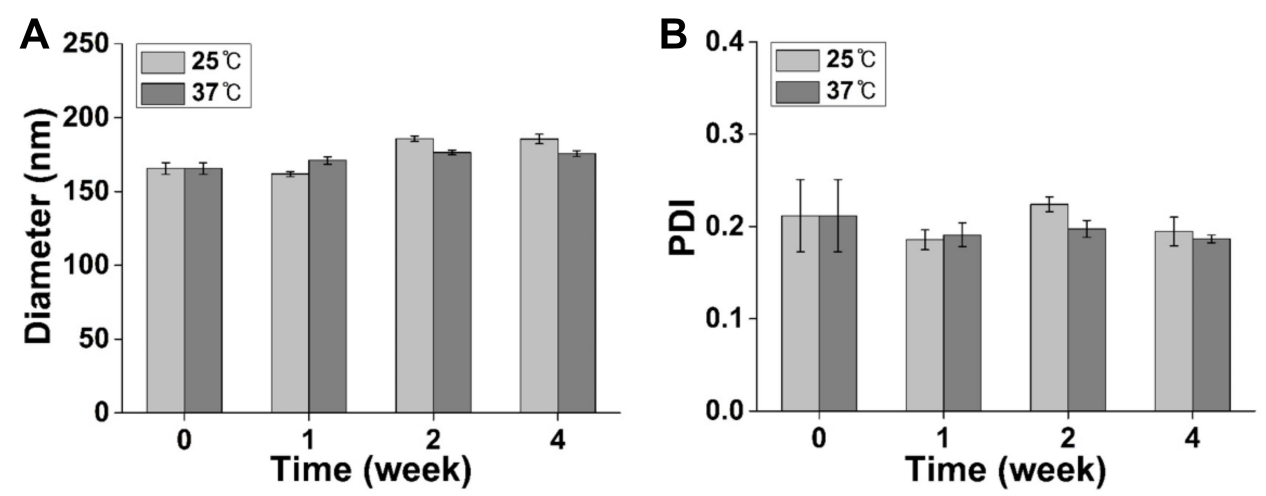

Figure 5 Stability of physicochemical properties of astaxanthin-loaded lecithin nano-liposol (ASTA@Lec NS; I5 wt\%) after 0-4 weeks of storage at room temperature and body temperature. (A) Hydrodynamic diameters; (B) polydispersity index (PDI) of ASTA@Lec NS (I5 wt\%) after 0-4 weeks of storage.

Abbreviation: PDI, polydispersity index.
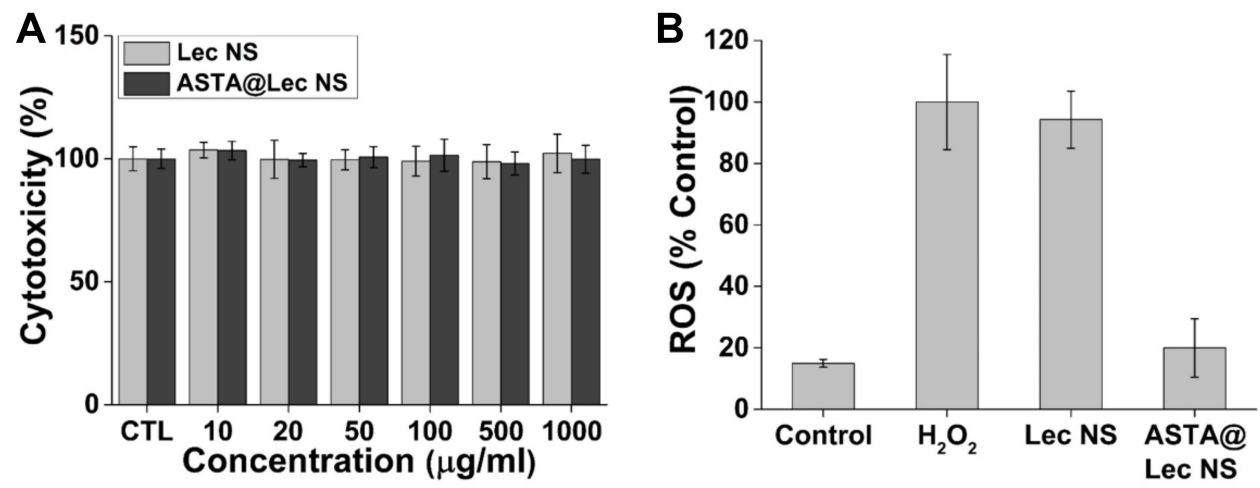

Figure 6 In vitro assays using CCK-8 and H2DCFDA assay kit. (A) Cytotoxicity analysis of lecithin nano-liposol (Lec NS) and astaxanthin-loaded lecithin nano-liposol (ASTA@Lec NS; 15 wt\%). (B) Antioxidant activity of Lec NS and ASTA@Lec NS (I5 wt\%), while the control and $\mathrm{H}_{2} \mathrm{O}_{2}$ samples indicate the lowest and highest ROS level. Abbreviations: Lec NS, lecithin nano-liposol; ASTA@Lec NS, astaxanthin-loaded lecithin nano-liposol; ROS, reactive oxygen species; $\mathrm{H}_{2} \mathrm{O}_{2}$, hydrogen peroxide.

indicates that ASTA@Lec NS is biocompatible and does not cause any harmful effects.

ASTA@Lec NS exhibited significant antioxidant activity at low concentrations of $10 \mu \mathrm{g} / \mathrm{mL}$ (Figure 6B). Following treatment with $\mathrm{H}_{2} \mathrm{O}_{2}$ as an oxidative stress agent, DCF fluorescence was analyzed to quantify in vitro level of ROS. NIH $3 \mathrm{~T} 3$ cells treated with only $\mathrm{H}_{2} \mathrm{O}_{2}$ was normalized as $100 \%$ ROS levels. The encapsulating material (Lec NS) alone, barely reduced ROS levels with fluorescence being approximately 94\%. In contrast, ASTA@Lec NS effectively scavenged cellular ROS to a level of approximately $20 \%$, which similar to that of the negative control at approximately 15\%. This proved that ASTA@Lec NS possessed very high antioxidant activity, both in situ and in vitro.

\section{In vitro Wound Healing Activity of ASTA@Lec NS}

Elimination of excessive ROS prevents endothelial dysfunction, damage to vessel walls, neurological diseases, and other negative outcomes. ${ }^{40,41}$ In particular, the scavenging activity and bio-signaling induced by ASTA may play a key role in wound healing. Scratch wound-healing assays are widely accepted as a direct and inexpensive in vitro wound healing test, alternatives to testing on animals. ${ }^{15}$ After forming a scratch in the cell monolayer, cells around the wound attempt to restore the damaged area through cell proliferation and migration. We analyzed the wound healing activity of ASTA@Lec NS by evaluating its effect on cell growth (Figure 7). Following $24 \mathrm{~h}$ of treatment, the scratch distance of the control group without ASTA@Lec NS exhibited almost no change from that of the initial distance of $321 \pm 7 \mu \mathrm{m}$ (Figure 7A). However, the scratch distance of the ASTA@Lec NS-treated cells decreased from $324 \pm 6 \mu \mathrm{m}$ to $227 \pm 19 \mu \mathrm{m}$ after $24 \mathrm{~h}$ of treatment. The effect of wound healing increased relative to ASTA concentration (Figure 7B); specifically, the scratch distances of cells treated with ASTA@Lec NS at different concentrations $(10,100,500$, and $1000 \mu \mathrm{g} / \mathrm{mL})$ were approximately $328 \mu \mathrm{m}, 291 \mu \mathrm{m}, 254 \mu \mathrm{m}$, and $227 \mu \mathrm{m}$, 

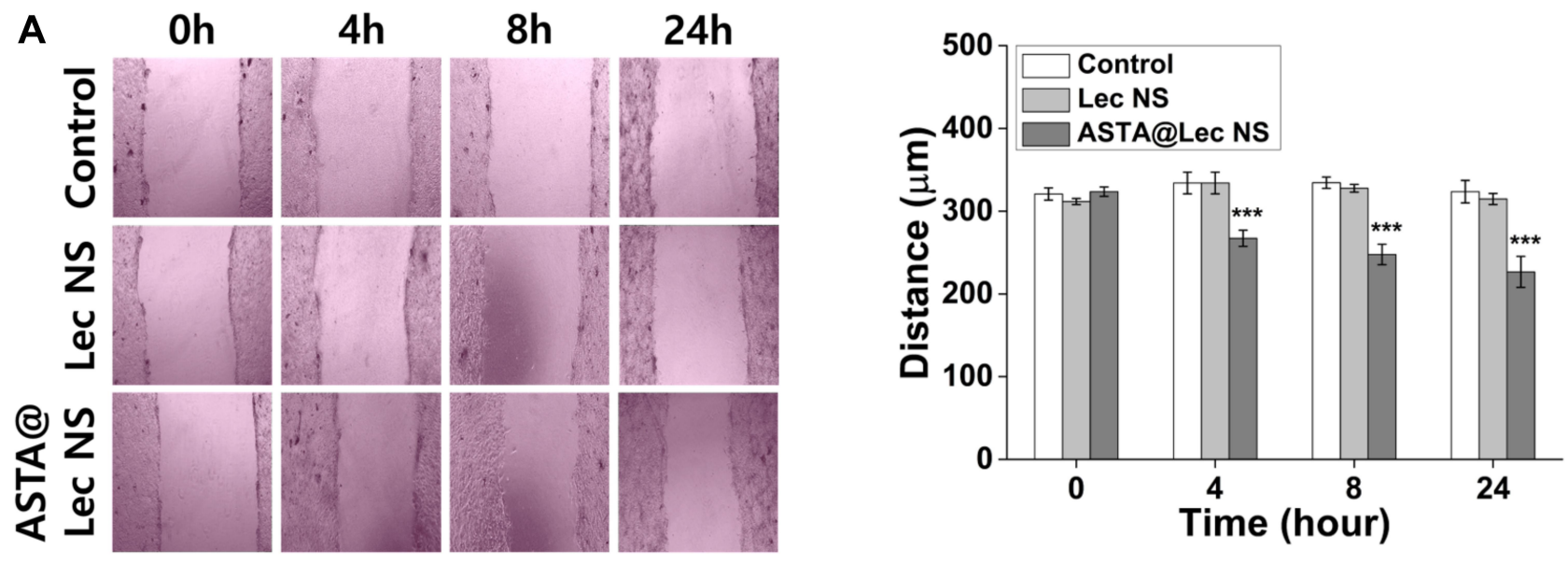

B
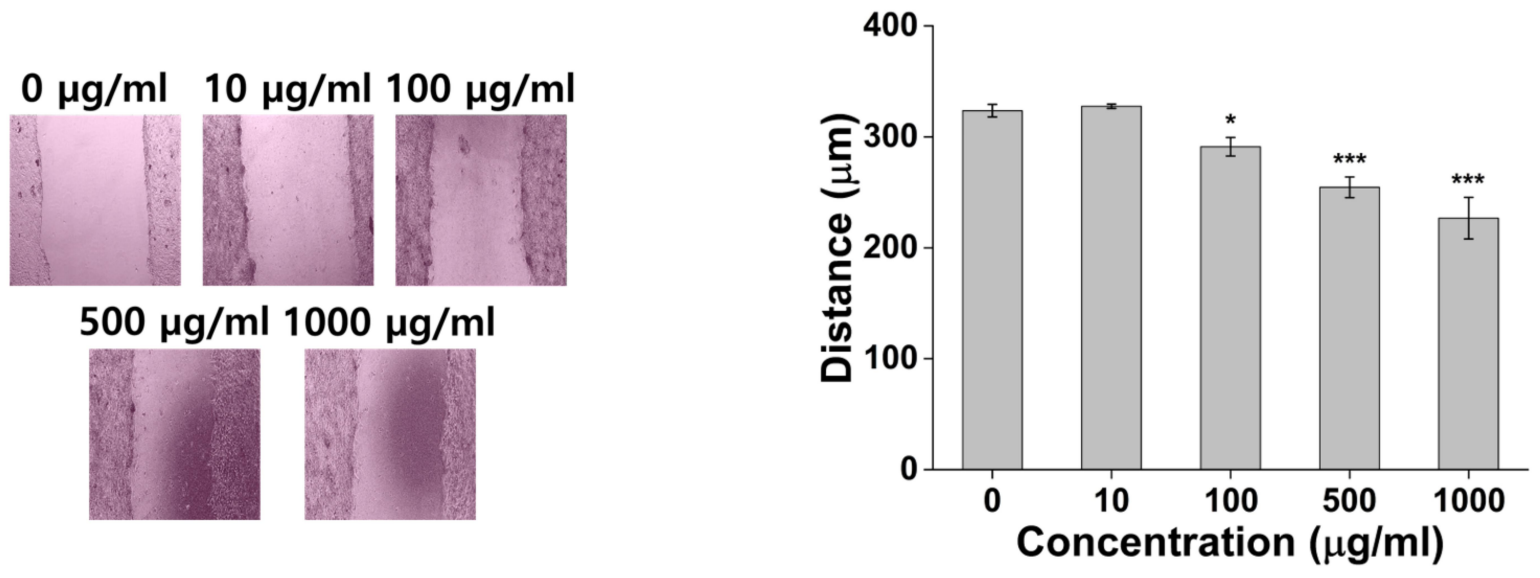

Figure 7 In vitro wound healing activity of astaxanthin-loaded lecithin nano-liposol (ASTA@Lec NS; I5 wt\%). (A) Wound closure of NIH 3T3 fibroblast cells after treatment of lecithin nano-liposol (Lec NS) and ASTA@Lec NS for I d. (B) Wound healing activity of ASTA@Lec NS at different concentrations after 24 h of treatment. Notes: The p-value below 0.05 or 0.001 was denoted by * or ***, respectively.

Abbreviations: Lec NS, lecithin nano-liposol; ASTA@Lec NS, astaxanthin-loaded lecithin nano-liposol.

respectively. Wound closure following a treatment of $1000 \mu \mathrm{g} /$ mL of ASTA@Lec NS was statistically greater than that of the control group $(p<0.001)$. This is consistent with the report by Chou et al, that ASTA has a positive effect on skin wound healing by promoting collagen generation in human dermal fibroblasts and inhibiting expression of matrix metalloproteinases MMP-1 and MMP-3. ${ }^{42}$

\section{Conclusion}

In this study, ASTA@Lec NS was successfully developed using a simple emulsion evaporation method. Hydrodynamic diameter, surface charge, and morphology were characterized forASTA@Lec with different LC. Without any change in its physicochemical properties, it could load high amount of ASTA with high efficiency. Furthermore, ASTA@Lec NS was stable for a sufficiently long period and readily lyophilized to powder state when an appropriate amount of sucrose was used as a cryopreservative. It suggests a feasibility of storage and transport for its various applications. In addition, the enhanced aqueous solubility of ASTA@Lec largely improved the bioavailability of ASTA. As a result, it could remove ROS and promote wound healing of NIH 3T3 cells more effectively without causing any cytotoxicity. Therefore, ASTA@Lec NS represents an outstanding delivery system to overcome limitations of ASTA for its applications in functional foods, medicines, cosmetics, and many others.

\section{Acknowledgment}

This research was supported by the National Research Foundation of Korea (NRF) grant No. NRF-2018 R1D1A1B07043620 (W.I.C.) funded by the Korea government (MSIT) and the Soonchunhyang University Research Fund (J.-M.L.). 


\section{Disclosure}

The authors report no conflicts of interest in this work.

\section{References}

1. Ambati RR, Phang SM, Ravi S, Aswathanarayana RG. Astaxanthin: sources, extraction, stability, biological activities and its commercial applications - a review. Mar Drugs. 2014;12(1):128-152. doi:10.3390/md12010128

2. Stahl W, Sies H. Bioactivity and protective effects of natural carotenoids. Biochim Biophys Acta. 2005;1740(2):101-107. doi:10.1016/j.bbadis.2004.12.006

3. Affandi MMM, Julianto T, Majeed A. Development and stability evaluation of astaxanthin nanoemulsion. Asian $J$ Pharm Clin Res. 2011;4(1):142-148.

4. Hama S, Takahashi K, Inai Y, et al. Protective effects of topical application of a poorly soluble antioxidant astaxanthin liposomal formulation on ultraviolet-induced skin damage. J Pharm Sci. 2012;101(8):2909-2916. doi:10.1002/jps.23216

5. Zuluaga M, Gueguen V, Letourneur D, Pavon-Djavid G. Astaxanthinantioxidant impact on excessive reactive oxygen species generation induced by ischemia and reperfusion injury. Chem Biol Interact. 2018;279:145-158. doi:10.1016/j.cbi.2017.11.012

6. Singh KN, Patil S, Barkate H. Protective effects of astaxanthin on skin: recent scientific evidence, possible mechanisms, and potential indications. J Cosmet Dermatol. 2020;19(1):22-27. doi:10.1111/ jocd. 13019

7. Susumu H, Sachiko U, Asako Y, et al. Scavenging of hydroxyl radicals in aqueous solution by astaxanthin encapsulated in liposomes. Biol Pharm Bull. 2012;35(12):2238-2242. doi:10.1248/bpb.b12-00715

8. Chen Y, Kao C, Huang H, et al. Astaxanthin reduces MMP expressions, suppresses cancer cell migrations, and triggers apoptotic caspases of in vitro and in vivo models in melanoma. $J$ Funct Foods. 2017;31:20-31. doi:10.1016/j.jff.2017.01.005

9. Shimokawa T, Yoshida M, Fukuta T, Tanaka T, Inagi T, Kogure K. Efficacy of high-affinity liposomal astaxanthin on up-regulation of age-related markers induced by oxidative stress in human corneal epithelial cells. J Clin Biochem Nutr. 2019;64(1):27-35. doi:10.3164/jcbn.18-27

10. Wu YC, Huang HH, Wu YJ, Manousakas I, Yang CC, Kuo SM. Therapeutic and protective effects of liposomal encapsulation of astaxanthin in mice with alcoholic liver fibrosis. Int $J$ Mol Sci. 2019;20(16):4057-4082. doi:10.3390/ijms20164057

11. Ritto D, Tanasawet S, Singkhorn S, et al. Astaxanthin induces migration in human skin keratinocytes via Racl activation and RhaoA inhibition. Nutr Res Pract. 2017;11(4):275-280. doi:10.4162/nrp.2017.11.4.275

12. Meephansan J, Rungjang A, Yingmema W, Deenonpoe R, Ponnikorn S. Effect of astaxanthin on cutaneous wound healing. Clin Cosmet Investig Dermatol. 2017;13(10):259-265. doi:10.2147/ CCID.S142795

13. Kim J, Lee CM. Anti-inflammatory effects and influence on fibroblast growth of astaxanthin-cyclodextrin nanoparticles. $J$ Chitin Chitosan. 2018;23(3):170-175. doi:10.17642/jcc.23.3.5

14. Roy S, Khanna S, Sen CK. Redox regulation of the VEGF signaling path and tissue vascularization: hydrogen peroxide, the common link between physical exercise and cutaneous wound healing. Free Radic Biol Med. 2008;44(2):180-192. doi:10.1016/j.freeradbiomed.2007.01.025

15. Schieber M, Chandel NS. ROS function in redox signaling and oxidative stress. Curr Biol. 2014;24(10):R453-462. doi:10.1016/j. cub.2014.03.034

16. Christophersen AG, Jun H, Jorgensen K, Skibsted LH. Photobleaching of astaxanthin and canthaxanthin. Quantum-yields dependence of solvent, temperature, and wavelength of irradiation in relation to packaging and storage of carotenoid pigmented salmonoids. Z Lebensm Unters Forsch. 1991;192(5):433-439. doi:10.1007/BF01193143
17. Sangsuriyawong A, Limpawattana M, Siriwan D, Klaypradit W. Properties and bioavailability assessment of shrimp astaxanthin loaded liposomes. Food Sci Biotechnol. 2018;28(2):529-537. doi:10.1007/s10068-018-0495-x

18. Rao AR, Sarada R, Ravishankar GA. Stabilization of astaxanthin in edible oils and its use as an antioxidant. J Sci Food Agric. 2007;87 (6):957-965. doi:10.1002/jsfa.2766

19. Tachaprutinun A, Udomsup T, Luadthong $C$, Wanichwecharungruang S. Preventing the thermal degradation of astaxanthin through nanoencapsulation. Int J Pharm. 2009;374(1-2):119-124. doi:10.1016/j.ijpharm.2009.03.001

20. Kaga K, Honda M, Adachi T, et al. Nanoparticle formation of PVP/ astaxanthin inclusion complex by solution-enhanced dispersion by supercritical fluids (SEDS): effect of PVP and astaxanthin Z-isomer content. J Supercrit Fluids. 2018;136:44-51. doi:10.1016/j.supflu.2018.02.008

21. Shen Q, Quek SY. Microencapsulation of astaxanthin with blends of milk protein and fiber by spray drying. $J$ Food Eng. 2014;123:165-171. doi:10.1016/j.jfoodeng.2013.09.002

22. Chen X, Chen R, Guo Z, Li C, Li P. The preparation and stability of the inclusion complex of astaxanthin with $\beta$-cyclodextrin. Food Chem. 2007;101(4):1580-1584. doi:10.1016/j.foodchem.2006.04.020

23. Yuan C, Du L, Jin Z, Xu X. Storage stability and antioxidant activity of complex of astaxanthin with hydroxypropyl- $\beta$-cyclodextrin. Carbohydr Polym. 2013;91(1):385-389. doi:10.1016/j.carbpol.2012.08.059

24. Ruiz VR, Dorado JAS, Barzegari A, et al. Astaxanthin-loaded nanostructured lipid carriers for preservation of antioxidant activity. Molecules. 2018;23(10):2601-2612. doi:10.3390/molecules23102601

25. Suntres ZE. Liposomal antioxidants for protection against oxidant-induced damage. $J$ Toxicol. 2011;2011(2):152474-152489. doi:10.1155/2011/152474

26. Anarjan N, Mirhosseini H, Baharin BS, Tan CP. Effect of processing conditions on physicochemical properties of astaxanthin nanodispersions. Food Chem. 2010;123(2):477-483. doi:10.1016/j. foodchem.2010.05.036

27. Ribeiro HS, Cruz RCD. Highly concentrated carotenoid-containing emulsions. Eng Life Sci. 2005;5(1):84-88. doi:10.1002/ elsc. 200403367

28. Wang Y, Li P, Tran TT, Zhang J, Kong L. Manufacturing techniques and surface engineering of polymer based nanoparticles for targeted drug delivery to cancer. Nanomaterials. 2016;6(2):26-44. doi:10.3390/nano6020026

29. Na Y, Lee JS, Woo J, et al. Reactive oxygen species (ROS)-responsive ferrocene-polymer-based nanoparticles for controlled release of drugs. J Mater Chem B. 2020;8(9):1906-1913. doi:10.1039/C9TB02533B

30. Pan L, Zhang S, Gu K, Zhang N. Preparation of astaxanthin-loaded liposomes: characterization, storage stability and antioxidant activity. Cyta J Food. 2018;16(1):607-618. doi:10.1080/19476337.2018.1437080

31. Chintong S, Phatvej W, Rerk-Am U, Waiprib Y, Klaypradit W. In vitro antioxidant, antityrosinase, and cytotoxic activities of astaxanthin from shrimp waste. Antioxidants. 2019;8(5):128-138. doi:10.3390/antiox 8050128

32. Apak R, Gorinstein S, Böhm V, Schaich KM, Özyürek M, Güçlü K. Methods of measurement and evaluation of natural antioxidant capacity/activity (IUPAC technical report). Pure Appl Chem. 2013;85 (5):957-998. doi:10.1351/PAC-REP-12-07-15

33. Ausborn M, Schreier H, Brezesinski G, Fabian H, Meyer HW, Nuhn P. The protective effect of free and membrane-bound cryoprotectants during freezing and freeze-drying of liposomes. $J$ Control Release. 1994;30(2):105-116. doi:10.1016/0168-3659(94)90257-7

34. Xu H, Chen P, Wang L, et al. Skin-permeable liposome improved stability and permeability of bFGF against skin of mice with deep second degree scald to promote hair follicle neogenesis through inhibition of scar formation. Colloids Surf B Biointerfaces. 2018;172:573-585. doi:10.1016/j.colsurfb.2018.09.006 
35. Wan F, Zhang C, Jin Q, et al. Protective effects of astaxanthin on lipopolysaccharide-induced inflammation in bovine endometrial epithelial cells. Biol Reprod. 2020;102(2):339-347. doi:10.1093/ biolre/ioz187

36. Oh H, Lee JS, Sung D, et al. Synergistic antioxidant activity of size controllable chitosan-templated Prussian blue nanoparticle. Nanomedicine. 2019;14(19):2567-2578. doi:10.2217/nnm-2019-0223

37. Satish A, Korrapati PS. Fabrication of triiodothyronine incorporated nanofibrous biomaterial: its implications on wound healing. RSC $A d v$. 2015;5(102):83773-83780. doi:10.1039/C5RA14142G

38. Li J, Sun W, Ramaswamy HS, et al. High pressure extraction of astaxanthin from shrimp waste (Penaeus vannamei boone): effect on yield and antioxidant activity. J Food Process Eng. 2016;40(2): e12353. doi:10.1111/jfpe. 12353

39. Winden ECA, Crommelin DJA. Long term stability of freeze-dried, lyoprotected doxorubicin liposomes. Eur J Pharm Biopharm. 1997;43(3):295-307.
40. Cloonan L, Fitzpatrick KM, Kanakis AS, Furie KL, Rosand J, Rost NS. Metabolic determinants of white matter hyperintensity burden in patients with ischemic stroke. Atherosclerosis. 2015;240 (1):149-153. doi:10.1016/j.atherosclerosis.2015.02.052

41. Lee D, Lee YJ, Lee YJ. Astaxanthin protects against MPTP/MPP +-induced mitochondrial dysfunction and ROS production in vivo and in vitro. Food Chem Toxicol. 2011;49(1):271-280. doi:10.1016/j. fct.2010.10.029

42. Chou H, Lee C, Pan J, et al. Enriched astaxanthin extract from Haematococcus pluvialis augments growth factor secretions to increase cell proliferation and induces MMP1 degradation to enhance collagen production in human dermal fibroblasts. Int $\mathrm{J} \mathrm{Mol} \mathrm{Sci}$. 2016;17(6):955-967. doi:10.3390/ijms17060955

\section{Publish your work in this journal}

The International Journal of Nanomedicine is an international, peerreviewed journal focusing on the application of nanotechnology in diagnostics, therapeutics, and drug delivery systems throughout the biomedical field. This journal is indexed on PubMed Central, MedLine, CAS, SciSearch ${ }^{\mathbb{R}}$, Current Contents ${ }^{\mathbb{R}} /$ Clinical Medicine, $^{-}$
Journal Citation Reports/Science Edition, EMBase, Scopus and the Elsevier Bibliographic databases. The manuscript management system is completely online and includes a very quick and fair peer-review system, which is all easy to use. Visit http://www.dovepress.com/ testimonials.php to read real quotes from published authors. 